\title{
Image du mort, effigie de l'ancêtre
}

The Image of the Deceased, the Effigy of the Ancestor (Dagara, Burkina Faso)

\section{Evariste Poda}

\section{OpenEdition}

\section{Journals}

Édition électronique

URL : http://journals.openedition.org/span/1236

DOI : $10.4000 /$ span. 1236

ISSN : 2268-1558

\section{Éditeur}

École pratique des hautes études. Sciences humaines

\section{Édition imprimée}

Date de publication : 1 décembre 1991

Pagination : 91-102

ISSN : 0294-7080

\section{Référence électronique}

Evariste Poda, «Image du mort, effigie de l'ancêtre », Systèmes de pensée en Afrique noire [En ligne], 11 | 1991, mis en ligne le 14 novembre 2013, consulté le 03 mai 2019. URL : http:// journals.openedition.org/span/1236 ; DOI : 10.4000/span.1236 
par

\section{Evariste Poda}

Un Dagara' ne boit pas sans avoir d'abord versé sur le sol quelques gouttes de sa boisson; il ne mange pas avant d'avoir laissé à terre une petite part de son repas: ces quelques gouttes, ces miettes de repas sont, dit-il, "pour les ancêtres". Dans les circonstances heureuses comme dans les heures sombres de sa vie, c'est vers eux encore que se tourne sa pensée. Comme en beaucoup d'autres populations d'Afrique occidentale, la référence aux ancêtres est chez les Dagara permanente et imprègne les moindres gestes de la vie quotidienne. Ce "dialogue continu" (Ilboudo, 1966) avec les ancêtres est si familier aux africanistes qu'il est peu de chercheurs qui s'en étonnent. Il y a bien pourtant quelque chose d'étrange dans le fait de s'adresser à un disparu et cela, d'une manière telle, qu'il soit pour tout un chacun "présent".

$\mathrm{Au}$ terme des rites funéraires qui permettent au défunt de quitter définitivement le monde des vivants et de rejoindre le "lieu des ancêtres", une statuette en bois est. ajoutée à l'ensemble des statuettes qui forme l'autel où sont invoqués les ancêtres d'un lignage (ou segment de lignage). Bien qu'elle partage quelques "traits" avec le disparu (son nom, son sexe, sa silhouette plus ou moins élancée ou trapue) cette statuette, appelée kpi daa ("bois de l'ancêtre") n'est pas une

\footnotetext{
' Les Dagara qui sont environ 250000 au Burkina Faso sont inégalement répartis dans trois provinces du sud et du sud-ouest du pays. Nos enquêtes ont été menées dans deux sous-groupes dagara: les Dagara Lobr et les Dagara Wiilé. Ils parlent la même langue (avec une différence portant sur l'alternance vocalique) et présentent la même organisation sociale.
} 
représentation du mort mais le signe d'une présence permanente, parmi les vivants, de cette instance que les Dagara appellent kpin et que, dans la littérature ethnologique, l'on appelle ancêtre. C'est du moins, nous allons le voir, ce qui ressort de l'examen de quelques séquences des rites funéraires.

\section{L'entré en deuil}

Dès l'annonce du décès, toute activité rituelle de type sacrificiel est suspendue dans le lignage du défunt (et cela, tant dans le segment résidant dans le village du défunt que dans ceux des villages voisins). Les sacrifices ne pourront reprendre qu'au lendemain d'un rite spécifique sur lequel nous allons revenir. Cette suspension des rites sacrificiels, les Dagara l'expliquent par l'état de "saleté" ou de "souillure" (degr qui dérive de deg "sale") dans lequel la mort de l'un de ses membres a placé tout le lignage: les larmes versées par la famille en deuil sont, disent-ils, le signe d'un dérèglement interne au lignage qui éloigne les ancêtres de leurs descendants.

Alors que le cadavre est encore présent, exposé sur une sorte de catafalqueà l'extérieur de l'enclos, l'on procède à un premier traitement des proches parents du défunt (conjoint et enfants): la tête des veuves (ou des veufs) est rasée et l'on place autour des reins de la veuve une ceinture en chanvre (hibiscus cannabinus); on attache à la cheville gauche de chacun des orphelins une cordelette de fibre, puis l'on passe à leur cou un collier fait d'une ficelle également de fibre sur laquelle est enfilé un cauri. Dès cet instant, la veuve doit adopter une conduite spécifique et respecter un certain nombre d'interdits (elle ne peut, notamment, changer de vêtement et doit s'asseoir à même la terre, les jambes allongées ) ; un veuf est soumis aux mêmes contraintes mais, à la différence de la veuve, il reçoit un bâton (qu'il ne pourra abandonner avant la fin des rites) et une peau d'animal (chèvre ou mouton) sur laquelle il doit s'asseoir.

Le veuf, comme la veuve, sera astreinte à ces conduites de deuil jusqu'à la fin des rites funéraires. Le rasage de la tête n'est qu'un premier rasage: ils en subiront un autre, les cérémonies qui viennent 
marquer les deux temps forts des funérailles (période intermédiaire et fin du deuil) étant à chaque fois fonction de la repousse de leurs cheveux.

Théoriquement, les enfants du mort sont, eux aussi, censés porter les cordelettes qui marquent leur état d'orphelin jusqu'au rite de levée de deuil. Néamoins, un parent à plaisanterie peut décider, au bout de quelques jours, que le moment est venu pour eux de "jeter la cendre sur le deuil"; il se chargera alors de détacher les cordelettes (en aucun cas, les orphelins ne peuvent enlever eux-mêmes ces signes de deuil, c'est l'objet d'un interdit).

La nuit qui suit l'enterrement, ainsi que les deux ou trois nuits suivantes, des proches parent(e)s et ami(e)s du survivant du couple se rendent à la maison du défunt pour participer à une cérémonie appelée ko yir gaa (de kuor "funérailles", yir "maison" et gaa "dormir"). Cette cérémonie dont le nom signifie littéralement "dormir à la maison des funérailles" consiste pour l'essentiel en un rassemblement auprès du conjoint pour lui porter réconfort et l'assurer de la compassion des siens.

Quelques jours plus tard, le doyen du lignage en deuil convoque une assemblée villageoise. Au cours de cette réunion, appelée kpi dio ("la chambre de l'ancêtre"), ceux envers qui le défunt avait de son vivant contracté une dette sont invités à se faire connâtre et les membres de la famille du mort s'engagent publiquement à les rembourser.

\section{Le temps du deuil}

Dans cette période intermédiaire qui court entre les rites d'enterrement et la levée du deuil prend place une cérémonie complexe qui, dans le temps même où elle marque la reprise de l'activité sacrificielle du lignage en deuil, signale à tous que la veuve (ou le veuf) entre dans une phase nouvelle de son deuil.

L'on repèrera, dans cette cérémonie, deux séquences rituelles bien distinctes par leur visée apparente, mais qui sont néanmoins étroitement associées l'une à l'autre par la nature du repère temporel choisi pour leur accomplissement, à savoir pour la première séquence dite 
$k p i ̃$ pir, la repousse des cheveux de la veuve (ou du veuf) et, pour la seconde séquence, appelée ko dan tuo, le second rasage de la tête de la veuve (ou du veuf).

La célébration du rite kpi pir (de kpĩn "ancêtre" et piru, terme formé sur le verbe piri "balayer, purifier"), dont le nom signifie littéralement "balayer les ancêtres", met fin à l'interdiction qui, depuis l'annonce du décès de l'un des leurs, frappaient les membres du lignage en les obligeant à différer tous les sacrifices destinés à leurs ancêtres. Cette cérémonie réunit dans la maison en deuil tous les doyens du lignage du défunt, ceux qui appartiennent au même segment villageois que ce dernier ainsi que tous ceux des segments installés dans des villages voisins. Rassemblés en cette pièce qui, comme en tous les enclos, est réservée aux autels d'ancêtre, ces doyens, munis de branchages peu feuillus d'une variété de figuier, viennent, chacun à leur tour, balayer par trois fois les "pieds" des statuettes représentant les ancêtres. Ces gestes sont accompagnés de paroles et d'invocations.

Cette reprise du "dialogue" avec les ancêtres de la maison en deuil (et partant avec ceux du lignage) précède et permet l'accomplissement de la séquence suivante centrée sur un second traitement de la veuve (ou du veuf).

Cette séquence, dont la dénomination ko dan tuo (de kuor "funérailles", daan "bière de mil" et tuo "amer") signifie littéralement "la bière amère des funérailles", vient scander ce temps des rites funéraires où le conjoint du mort franchit une nouvelle étape dans le "travail du deuil". La bière de mil préparée pour le rite de rasage des cheveux de la veuve (ou du veuf) est qualifiée "d'amère" car, comme celle utilisée dans les sacrifices faits aux ancêtres, elle ne peut être bue que par les initiés. Mais cette qualification vient aussi signifier qu'à cette phase du procès rituel, l'on est en un temps bien différent du temps de la levée de deuil, marqué, lui, par une cérémonie dont l'un des noms est ko dan maan, "la bière fraîche, calme, des funérailles".

Lorsque la "bière amère" est prête, la veuve (ou le veuf) est rasée, tout son corps est enduit d'une boue d'argile blanche (yag ra) et on lui attache autour de la tête une nouvelle cordelette de fibre. Ce rite 
porte le nom de yag ra guoru ("marque, trace d'argile"). Selon le mythe cosmogonique dagara, Nanmwine (Dieu) s'est servi de l'argile blanche yag ra pour fabriquer le corps humain. Lorsqu'on enduit ainsi le conjoint du mort de cette boue blanche, disent les Dagara, l'on cherche à lui faire comprendre qu'à présent, son partenaire est retourné à cette matière première dont tous les êtres humains sont faits. Cette interprétation suggère l'idée qu'entre le type de traitement rituel réservé au conjoint du défunt et l'état supposé du défunt lui-même, la pensée symbolique dagara pose un lien étroit. Comme si par des conduites de deuil différenciées au cours du rituel, l'on cherchait à montrer le détachement progressif du mort du monde des survivants.

Le conjoint devra conserver sa couche d'argile pendant trois semaines au moins; au terme de ce délai, le chef de famille informera ses proches que le moment est venu de procéder à la levée définitive du deuil.

\section{La levée de deuil et l'ancestralisation}

Le rite de levée de deuil est dénommé de différentes façons. On peut l'appeler yaaru, terme formé à partir du verbe yaa qui signifie "enlever, ôter, se dévêtir", mais aussi "se désengager"; ou ko dan mar, "la bière fraîche des funérailles" (mar signifiant "ce qui s'est refroidi" est ici utilisé, nous l'avons dit, par opposition à tuo "amer", "chaud"). Dans certaines régions du pays dagara, ce rite est parfois encore appelé ko dan baara , "la dernière bière des funérailles" (baara est formé sur le verbe baari "finir").

La présence de deux catégories de parents est nécessaire à l'accomplissement de cette cérémonie: le fils aîné, d'une part, les ce ko taa dem, d'autre part.

Comme dans un grand nombre de populations "voltaïques", un homme ne pourra pas, à sa mort, accéder au statut d'ancêtre s'il n'a pas laissé des fils vivants, nés d'une épouse dont la compensation matrimoniale a été versée. Une institution - toujours en vigueur chez les Dagara Wiilé et dans une moindre mesure chez les Dagara Lobr - 
permet toutefois un aménagement de cette règle, en offrant la possibilité à un couple stérile (ou à un couple dont tous les enfants seraient morts) d'être néanmoins parents d'un enfant (d'un point de vue social et rituel) qui pourra, à leur mort, prendre en charge les rites qui les feront accéder au statut d'ancêtre. Cette institution, dite du bi dira (de bie "enfant" et dira terme formé sur le verbe di "manger"), est fondée sur l'échange entre deux frères de leurs premiers-nés (cet échange peut se réduire au "don" des premiers-nés d'un homme (garçon et fille) à celui de ses frères qui ne peut avoir d'enfant). Les enfants ainsi "échangés" ou "donnés" sont appréhendés comme les vrais fils (ou filles) de ceux à la charge desquels ils ont été confiés et, à la mort de leur père social ou de leur mère sociale, ils effectueront, tels des fils aînés et filles aînées, le rite que nous décrivons ci-dessous.

L'autre catégorie de parents impliquée dans la cérémonie de la "bière fraîche" renvoie à une forme d'assistance rituelle entre deux lignages - que l'on rencontre dans d'autres populations de la même aire culturelle. Le terme de $c \varepsilon$ ko taa dem (de ce "couper"; ko "donner"; taa qui exprime la réciprocité et dem, plur. de sob, "propriétaire") que l'on pourrait traduire par l'expression "ceux qui coupent l'un pour l'autre" est un terme réciproque qu'emploient les membres de deux lignages liés par cette assistance rituelle. Dans le contexte des rites funéraires, c'est à cette catégorie de parent qu'est réservé le délicat travail rituel de sculpter les statuettes d'ancêtre.

Quand le chef de famille réunit les membres du lignage pour les informer que le temps de la levée de deuil est arrivé, le fils aîné du défunt ou, le cas échéant, le bi dira, exprime son désir d'aider son père à rejoindre le séjour des ancêtres. Les ce ko taa dem sont alors avertis qu'ils auront bientôt à tenir leur rôle.

Le jour fixé, le fils aîné accompagné des ce ko taa dem part en brousse. C'est à lui qu'il revient de désigner l'arbre sur lequel sera prélevée la branche d'où le travail des ce ko taa dem fera surgir l'effigie de son père. L'essence de cet arbre varie selon les lignages: karité pour les uns, cailcédrat pour d'autres. L' espèce la plus estimée pour ce travail est appelée $k u$ mwsli nuu, littéralement "l'arbre dont la plus petite branche ne peut être cassée par la seule force du bras";

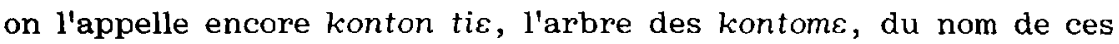


petits êtres de brousse qui, d'après certains mythes, ont transmis aux hommes les techniques (entre autres, le feu). Pour les membres d'un lignage déterminé, l'espèce choisie pour y tailler les effigies

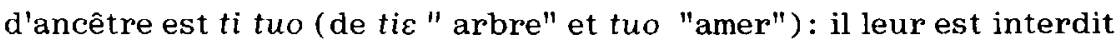
de prélever du bois de cette essence dans un but utilitaire (bois de chauffage, poutres des toits).

Une fois sélectionnée par le fils aîné, la branche est l'objet de grandes précautions. Pendant que l'un des $c \varepsilon$ ko taa dem la détache de l'arbre, le fils s'apprête à la recevoir. Elle ne doit pas tomber à terre. L'interdit est rigoureux: dès l'instant où elle est désignée, la branche est en effet perçue comme le corps même du défunt; qu'elle tombe au sol serait pour le fils commettre la même profanation que laisser tomber à terre le cadavre de son père. Ainsi délicatement réceptionnée par le fils, la branche peut ensuite être transmise au sculpteur (un autre ce ko taa dem) qui entame aussitôt le travail alors que le restant du groupe rentre au village.

A leur retour de brousse, les femmes commencent à préparer une

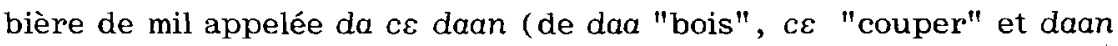
"bière"). Cette préparation, qui dure trois jours, rythme le travail de sculpture de l'effigie: il commence le même jour et doit être achevé dans les mêmes temps. Au soir des deux premiers jours, la statuette inachevée est cachée dans un buisson. De même que l'on cache aux regards le cadavre tant qu'il n'est pas lavé et habillé, on interdit à certaines catégories de gens (femmes et enfants) de voir l'effigie en cours de confection.

$\mathrm{Au}$ soir du troisième jour, la statuette entièrement sculptée est replacée dans les broussailles; seuls les ce ko taa dem savent où elle se trouve. Le chef de famille prend alors des vêtements du mort, $k u$ kpari (de ku "mort", "cadavre" et kpari "habits") et les remet aux enfants du défunt qui partent à la recherche de la statuette cachée. Quand ils la trouvent, ils la drapent dans les vêtements, avant de la ramener au village. Cette séquence du rite répète d'une certaine façon les gestes qui, au temps de l'enterrement, avaient précédé l'exposition du cadavre, quand les deuilleurs l'avaient revêtu d'un même genre de costume: un pantalon bouffant et une tunique ample 
aux longues et larges manches. Une fois la statuette trouvée et "habillée", une procession se forme derrière celui qui la porte. Le cortège se dirige vers le village. Les pleurs, alternés avec un chant de deuil, recommencent exactement comme aux premiers jours des funérailles. Arrivé à la maison du défunt, le cortège en fait trois fois le tour. La statuette est ensuite déposée dans une petite natte (seng bile) que l'on appuie contre la façade de la maison (dans certains lignages, elle est placée dans un poulailler). Les ce ko taa dem jettent des cauris sur la natte et son précieux contenu.

A cette étape du rite, l'effigie est appelée $k p i ́$ da pla, le "bois blanc de l'ancêtre ". Deux ou trois jours plus tard, quand elle sera installée sur l'autel familial et consacrée par un sacrifice sanglant, on l'appelera

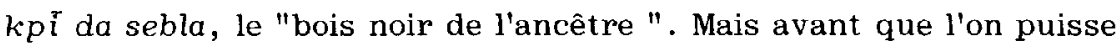
procéder à cette installation définitive de l'ancêtre dans la demeure de ses descendants, la veuve doit se soumettre à un dernier rite qui vient lui signifier que le temps de son deuil a pris fin.

Un vieillard du lignage des ce ko taa dem appelle la veuve qui s'est débarrassée des marques du deuil qu'elle portait jusqu'alors: elle s'est lavée et parée d'habits neufs. L'ancien prélève un peu de pâte de mil sur un plat préparé pour la circonstance, trempe la bouchée dans une sauce à base d'arachide et de viande et, s'adressant à la veuve, dit "je vais jeter ce morceau de nourriture aux pieds de ton époux. Y a-t-il quelque chose?". Si la réponse est négative, cela signifie que, depuis le décès de son conjoint, elle lui est restée fidèle. L'officiant jette alors la bouchée aux pieds de la statuette. Il répétera toute l'opération par trois fois. La veuve est, dès lors, définitivement libérée de son deuil et peut reprendre une vie normale. Mais, si à la question posée par l'ancien, elle répond par l'affirmative, celui-ci jettera le morceau de gâteau de mil vers le côté de la statuette et non à ses pieds. Chacun comprendra alors ce qui s'est produit. Cette femme est néanmoins libérée de son deuil, mais elle devra se soumettre à un rite de purification, faute de quoi elle prend le risque d'être frappée d'une maladie dont le nom, datura, est, selon certains informateurs, une déformation de l'expression daba nu taru signifiant "c'est l'homme qui la tient". 
Au lendemain de cette levée de deuil, l'on peut entamer les préparatifs du dernier rite de la longue série enclenchée par le décès d'un homme (ou d'une femme). Ce rite final est celui qui va permettre l'entrée dans la maison de la statuette qui, depuis son extraction de la brousse, reposait en un lieu intermédiaire aux abords de l'enclos (cour extérieure ou poulailler). De la bière de mil, à nouveau, doit être fabriquée. Lorsqu'au terme de trois jours elle est prête, le fils aîné ou, le cas échéant, le bi'dira transporte la statuette dans la petite pièce où se trouve l'autel familial, mais il ne la dépose pas immédiatement aux côtés des autres effigies d'ancêtre. Il doit d'abord offrir, selon ses moyens, un coq, un mouton, une chèvre ou un boeuf. I'animal est sacrifié et la statuette aspergée du sang de la victime. Le sacrificateur, qui est nécessairement l'un des ce ko taa dem, prend pour les membres de son lignage la moitié de la viande et remet l'autre à la famille. Cela fait, la statuette est intégrée à l'ensemble des effigies d'ancêtre qui forme l'autel. Un nouvel ancêtre est entré dans le panthéon familial, généralement érigé dans une petite case où seuls trois hommes peuvent entrer: le sacrificateur ( $s u o s o b$, le "propriétaire du couteau"), le récitant (no tu re) qui est le doyen du lignage et connaît tous les noms des ancêtres et, enfin, le "propriétaire de la corbeille" ( $k a \operatorname{sog} s o b$ ) qui possède le panier dans lequel sont amenées auprès de l'autel les volailles offertes en sacrifice.

Les séquences rituelles que nous venons de décrire appellent plusieurs remarques. L'on notera tout d'abord le rapport étroit qui s'y établit entre le traitement du conjoint survivant et les rites plus spécifiquement centrés sur le défunt. La détermination temporelle de ces derniers est à chaque fois, en effet, fonction d'une modification subie par le conjoint survivant en son corps (repousse des cheveux, rasage, bain qui le débarrasse de sa gangue de boue blanche). En liant ainsi étroitement le destin du conjoint survivant à celui de son partenaire défunt, les cérémonies funéraires dagara suggèrent l'idée que la transformation du conjoint au cours des différentes étapes qu'il parcourt accompagne, pour ne pas dire permet, celle du mort en ancêtre.

Le mode sur lequel est pensé cette dernière transformation apparait, selon nous, dans le curieux traitement en plusieurs temps du "bois 
de l'ancêtre". Nous repérerons trois moments bien distincts: $1^{\circ}$ ) un temps d'extraction (coupe de la branche, taille de l'effigie, transfert de la brousse au village); $2^{\circ}$ ) un temps intermédiaire (installation provisoire aux abords de la maison); $3^{\circ}$ ) un temps d'agrégation (installation définitive parmi les statuettes d'ancêtre). A ces trois moments correspondent des "attitudes" rituelles bien différentes. Nous avions déjà souligné la similitude que crée le rituel entre des gestes accomplis sur la dépouille au temps de l'enterrement et ceux dont est l'objet la statuette au temps de son extraction (la branche ne peut tomber à terre et doit être accueillie dans les bras du fils aîné du mort; tel le cadavre non préparé, elle est cachée aux regards tant que le travail de sculpture n'est pas achevé; une fois taillée, elle est emballée dans les "effets" du mort). Le changement de statut de l'effigie, nettement marqué par la distinction sémantique (et symbolique) que font les Dagara entre le "bois blanc" et le "bois noir", intervient très précisément, avons-nous dit, lorsque la statuette est pour la première fois support d'un sacrifice sanglant adressé à l'ancêtre. Traitée comme le corps du défunt avant qu'elle n'atteigne le village, mais perçue comme effigie de l'ancêtre une fois intégrée au panthéon, la statuette ne passe pas d'un statut à l'autre sans occuper une position intermédiaire où elle semble appréhendée tour à tour comme une représentation du mort et une effigie de l'ancêtre. On rappellera en effet que dans son adresse à la veuve, l'ancien désigne la statuette par l'expression "ton époux". Par contre, les pleurs et chants de deuil que déclenche l'arrivée du bois sculpté au village ne peuvent se comprendre si l'on maintient l'idée que ce qui se présente à ce moment-là aux regards de la famille, c'est une image du défunt. Il nous apparait que cette expression obligatoire des sentiments vient signifier, à chacun, que l'entrée de la statuette marque le départ définitif du défunt hors des murs de la maison. L'enchaînement des séquences rituelles renforce cette hypothèse. C'est bien, en effet, la présence du "bois blanc" dans la cour extérieure de la maison - et ce que cela implique d'une absence du défunt - qui permet au conjoint survivant d'être définitivement libéré des contraintes du deuil.

La statuette partage certains "traits" avec le défunt mais changés en symboles (un bois simple pour les femmes, un bois à deux branches pour les hommes, par exemple). De même, le traitement rituel vient 
"dire" la sorte de lien qui existe entre la statuette et l'image du défunt pour finalement ne conserver que l'idée de la rupture de ce lien. Il nous semble que pour construire l'ancêtre, le procès rituel mis en oeuvre par les Dagara fait appel à une dialectique de la présence et de l'absence.

Evariste Poda IRSSH - Ouagadougou

\section{Références bibliographiques}

Dialle, E.B.

1981 Le culte des ancêtres en pays mossi: le village de Moutti dans la sous-préfecture de Ziniaré. Dakar.

Goody, Jack

1977 "Mémoire et apprentissage dans les sociétés avec et sans écriture. La transmission du Bagré". L'homme, janviermars, pp. 29-52

Ilboudo, Pierre

1966 Croyances et pratiques religieuses traditionnelles des Mossi. Paris.

Somé, Magloire

1986 La christanisation du pays dagara 1929-1969. L'exemple de la paroisse de Daanû. Ouagadougou. 